\title{
Accuracy vs. Speed: Scalable Entity Coreference on the Semantic Web with On-the-Fly Pruning
}

\author{
Dezhao Song \\ Department of Computer Science and Engineering \\ Lehigh University \\ Bethlehem, PA 18015, USA \\ des308@lehigh.edu
}

\author{
Jeff Heflin \\ Department of Computer Science and Engineering \\ Lehigh University \\ Bethlehem, PA 18015, USA \\ heflin@cse.lehigh.edu
}

\begin{abstract}
One challenge for the Semantic Web is to scalably establish high quality owl:sameAs links between coreferent ontology instances in different data sources; traditional approaches that exhaustively compare every pair of instances do not scale well to large datasets. In this paper, we propose a pruning-based algorithm for reducing the complexity of entity coreference. First, we discard candidate pairs of instances that are not sufficiently similar to the same pool of other instances. A sigmoid function based thresholding method is proposed to automatically adjust the threshold for such commonality on-the-fly. In our prior work, each instance is associated with a context graph consisting of neighboring RDF nodes. In this paper, we speed up the comparison for a single pair of instances by pruning insignificant context in the graph; this is accomplished by evaluating its potential contribution to the final similarity measure. We evaluate our system on three Semantic Web instance categories. We verify the effectiveness of our thresholding and context pruning methods by comparing to nine stateof-the-art systems. We show that our algorithm frequently outperforms those systems with a runtime speedup factor of 18 to 24 while maintaining competitive F1-scores. For datasets of up to 1 million instances, this translates to as much as 370 hours improvement in runtime.
\end{abstract}

Keywords-Semantic Web, Entity Coreference, Linked Data, Scalability, Pruning

\section{INTRODUCTION}

The purpose of entity coreference is to decide which identifiers (e.g., person names, locations, etc.) refer to the same real world entity. This is an essential task when integrating data from multiple sources. Entity coreference in the Semantic Web is used to detect equivalent (coreferent) ontology instances. Minimally, an ontology is an explicit and formal specification of a conceptualization, formally describing a domain of discourse. An ontology consists of a set of terms (classes) and their relationships (class hierarchies and predicates). RDF is a graph based data model for describing resources and their relationships in the Web. The source $s$ of an edge, called its subject in $\mathrm{RDF}$, is an identifier (i.e., a URI). The target $o$ of an edge, called its object in RDF, can be either an identifier or a literal value, such as strings, numbers, etc. Each edge is labeled with a predicate $p$. Since an edge is represented by a tuple $\langle s, p, o\rangle$, they are called triples. A URI that takes the subject place in one triple can be the object in another. Note, since ontology instances are represented by URIs assigned in a decentralized fashion, syntactically distinct URIs may actually represent the same entity. For example, a researcher can have multiple distinct URI identifiers in DBLP, ACM and CiteSeer but such URIs actually represent the same real world person; thus they are coreferent. In the Semantic Web, coreferent instances are linked to each other via the owl:sameAs predicate.

One well-known effort related to entity coreference is Linked Data [1] that enables people to publish their data with links to existing datasets. According to the latest statistics of the Linked Open Data (LOD) cloud ${ }^{1}$, there are currently 295 datasets from various domains with more than 31 billion RDF triples and about 500 million links across different datasets. A traditional approach to handling this entity coreference problem is to exhaustively compare every pair of instances in a dataset [2]-[4], which could be extremely expensive for large-scale datasets. Therefore, there is the need to develop scalable entity coreference algorithms to be able to efficiently process large-scale datasets. Furthermore, Halpin et al. [5] reported that only $50 \%( \pm 21 \%)$ of the owl:sameAs links from the LOD Cloud are correct. This troubling fact emphasizes that although scalability is important, we must also achieve satisfying precision and recall.

In this paper, we propose a pruning-based algorithm for reducing the complexity of entity coreference within a dataset itself. Here, a "dataset" may contain comparable instances from multiple heterogeneous sources. For example, different datasets may use different terms to define the "Researcher" class and ontology instances of all such classes are comparable. First, we design an on-the-fly candidate selection technique to reduce the number of instance pairs to be computed. During the entity coreference process, each instance is compared against other instances; and we hypothesize that two coreferent instances should have similar matching histories, i.e., they should be similar to a sufficiently common set of other instances. We further propose a sigmoid function based thresholding method to automatically adjust the threshold on such history similarity in order to gain a good balance between runtime and F1-score. To speed up the computation for a single pair of instances, we evaluate the potential contribution of their context and only consider the context that is likely to make a significant contribution to their final similarity.

\footnotetext{
${ }^{1}$ http://www4.wiwiss.fu-berlin.de/lodcloud/state/
} 
We evaluate our system on three Semantic Web instance categories. By comparing to alternatives that only adopt a subset of our proposed techniques, we verify the effectiveness of each individual component. Through the comparison to 9 state-of-the-art systems, we show that our algorithm runs 18 to 24 times faster with comparably good precision and recall. A scalability test on up to 1 million instances shows that the runtime differences become even more substantial as we increase the size of the datasets.

We organize the rest of the paper as follows. Section II provides some background information. We then formally present our pruning-based algorithm in Section III and discuss related work in Section IV. We present our evaluations in Section V and conclude in Section VI.

\section{BACKGROUND}

We first introduce a basic entity coreference algorithm EPWNG [4], Exhaustive Pairwise Entity Coreference based on Weighted Neighborhood Graph. It computes the similarity between every instance pair in a dataset based on a set of paths, i.e., the context, as shown in Figure 1. A

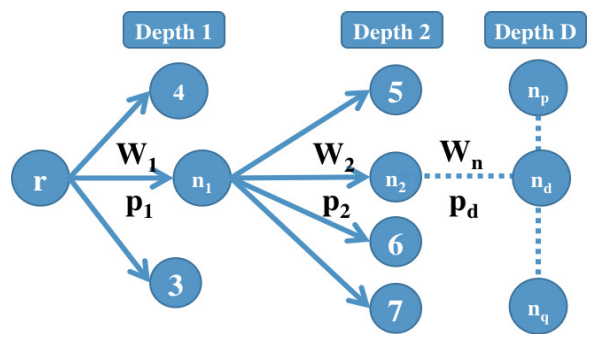

Figure 1. Weighted Neighborhood Graph $(G)$

path is defined as follows: path $=\left(r, p_{1}, n_{1}, \ldots, p_{d}, n_{d}\right)$, where $r$ is an ontology instance; $n_{i}$ and $p_{i}(i>0)$ are any expanded RDF node and predicate in the path. $N(G, r)$ denotes the context (a set of paths that start from $r$ and end on another RDF node) for $r$ in RDF graph $G$; End(path) gives the last node in a path. Each node $n_{i}$ has a weight $W_{i}$ computed based on the discriminability of its associated predicate $p_{i}$; path weight is the multiplication of all its node weights. When collecting context, we adopt breadthfirst search and eliminate cycles [4].

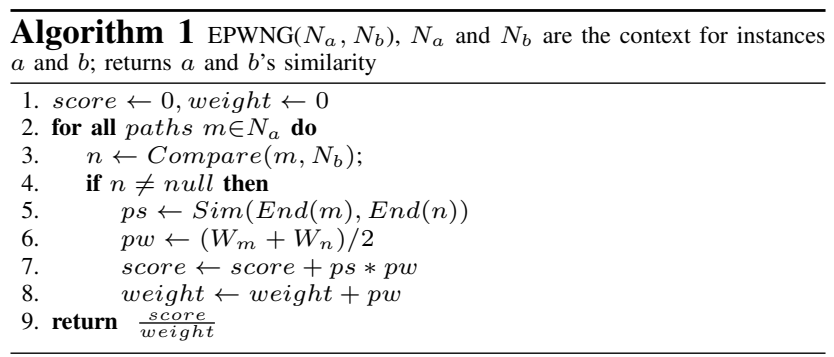

EPWNG (Algorithm 1) compares the comparable paths in the context of two instances $a$ and $b$. Two paths are comparable if their predicates at corresponding positions are comparable (determined by $P t C m p$ ), i.e., having the same semantics. For example, predicate CiteSeer:name is comparable to DBLP:name. Here, we created these

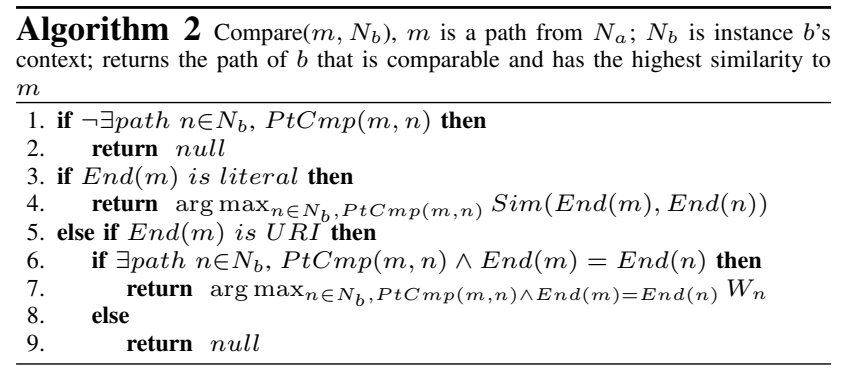

mapping axioms of predicate comparability manually; but we note that ontology alignment [6], a well studied topic in the Semantic Web, can help automatically determine predicate comparability across multiple ontologies, which is out of the scope of this paper. Sim calculates the string similarity between the last nodes of two paths. For each path $m$ of $a$, we call Compare() (see Algorithm 2) to choose a path $n$ of $b$ that is comparable to and has the highest similarity score to $m$, denoted as $p s$. The average weight of path $m\left(W_{m}\right)$ and $n\left(W_{n}\right)$ is used as the weight for $p s$. The process is repeated for every path of $a$. The weighted average on such (path score, path weight) pairs is computed as the final similarity score for $a$ and $b$.

There are two key factors that prevent $E P W N G$ from scaling to large-scale datasets. On one hand, EPWNG compares every pair of instances in a dataset thus making the entire entity coreference process for large datasets prohibitively expensive. So, one critical question is: Can we prune instance pairs that are unlikely to be coreferent to reduce the overall complexity? On the other hand, for two instances, $E P W N G$ compares all pairs of their comparable paths. Assuming context graphs have branching factor $n$ and depth $d$, the complexity for computing a single instance pair is $O\left(n^{2 d}\right)$, therefore making $E P W N G$ very time-consuming for handling large context. So, another interesting question here is: Can we only consider the context that could potentially make a significant contribution to the final similarity score between two instances to further speed up the process?

\section{Algorithm}

Algorithm 3 presents the pseudo-code of our proposed pruning-based entity coreference algorithm $P$-EPWNG. At line 1, we adopt a simple yet effective filtering method, i.e., we check if two instances share a single token in the literal values of their top $\mathrm{k} \%$ most disambiguating context, and if the cosine similarity of such instances' top $\mathrm{k} \%$ context is above a threshold $\delta$. First, two instances cannot be coreferent if they do not share at least one common word in their context; also, the cosine similarity is used to better handle contexts with many tokens. For example, for publication instances, their titles are generally very disambiguating and thus included in the top $\mathrm{k} \%$ context; however, only sharing a single token does not necessarily indicate that they could be coreferent in many cases.

From line 3 to 10 , we propose an on-the-fly candidate selection technique based upon instances' matching histories to effectively prune out instance pairs that are not 


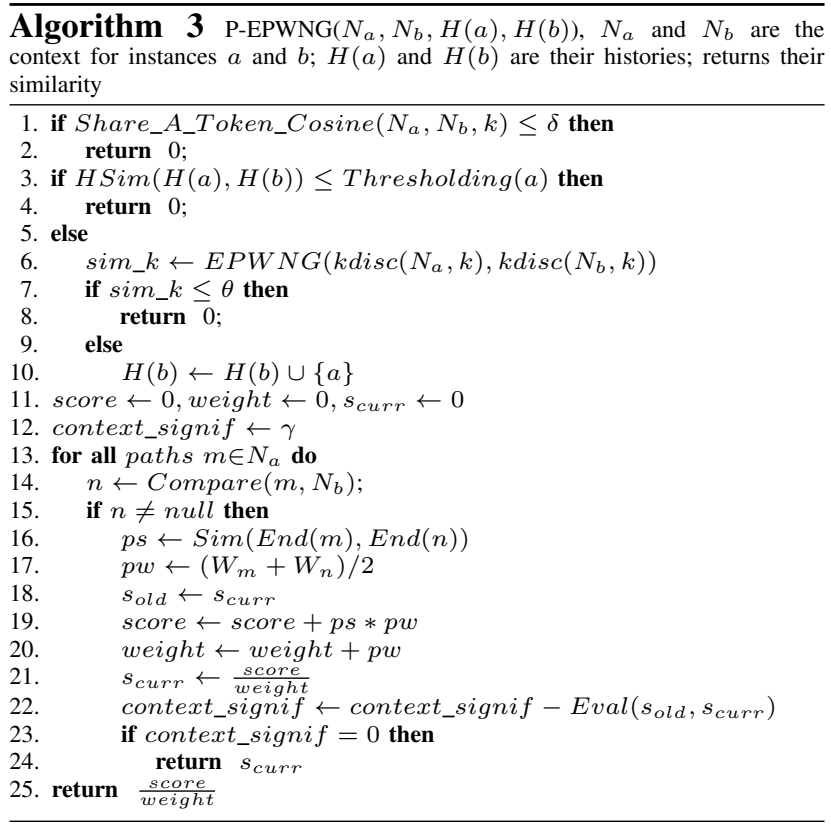

likely to be coreferent. Consider that during the entity coreference process, an instance is compared to many other instances; the results of these prior comparisons could be useful in determining whether two instances might be coreferent. At any point in time, each instance should have a set of other instances that it is somewhat similar to. We define a function $H(a)$ to denote the set of similar instances of instance $a$, i.e., the matching history. We hypothesize that two coreferent instances should share a sufficient amount of common instances in their histories. Therefore, the general idea behind our on-the-fly candidate selection technique is that before we actually compute the similarity for instances $a$ and $b$, we adopt a lightweight method to examine if their histories are similar enough, i.e., if $H \operatorname{Sim}(H(a), H(b))$ is above some threshold (line 3). Instead of utilizing a fixed threshold on HSim, we propose a sigmoid function based thresholding method that gradually increases the threshold at runtime until an upper bound is reached, since there is very little history to compare at the beginning. HSim and the thresholding method are further presented in Section III-A.

From line 6 to 10, after an instance pair passes the history check, we should then apply a more expensive method to measure their similarity, which can also be used to determine if we should add one instance to the history of another. In our algorithm, at line 6 , we make this decision by computing two instances' similarity by using the EPWNG algorithm only with their top $\mathrm{k} \%$ most disambiguating context (as returned by $k \operatorname{disc}\left(N_{a}, k\right)$ ). Passing the history check does not necessarily mean the two instances are coreferent and thus it might not make sense to use full context.

Furthermore, we propose a context pruning technique inspired by the idea of quiescence search in game playing. The basic idea is that more time should be spent refining heuristic evaluations when there appear to be significant fluctuations in the value. In this paper, we define an evaluation function to estimate if the remaining context would still make a significant contribution to the final similarity score of two instances. At line 12, we initialize context_signif (context significance level) with a positive integer $\gamma$ that can be interpreted as the maximum times that we allow a new path to provide only a small contribution to the final similarity score. Then, at line 22, an evaluation function Eval is used to check the difference between $s_{c u r r}$ and $s_{\text {old }}$, i.e., if considering one more path still makes a significant change to the similarity score between two instances. We will prune the rest of the context when context_signif reduces to 0. Eval is further discussed in Section III-B.

\section{A. History-based on-the-fly Candidate Selection}

A Modified Jaccard Similarity Measure. As shown in Algorithm 3, we compute the similarity (HSim, line 3) between the matching histories of two instances. In this paper, we adopt a modified version of the well-utilized Jaccard similarity measure as defined in Equation 1:

$$
H \operatorname{Sim}(H(a), H(b))=\left\{\begin{array}{lr}
+\infty, & H(a)=\emptyset \text { or } H(b)=\emptyset \text { (1a) } \\
\frac{|H(a) \cap H(b)|}{|H(a) \cup H(b)|}, & \text { otherwise (1b) }
\end{array}\right.
$$

where $H(a)$ represents the matching history of instance $a$, i.e., a set of other instances that $a$ is similar to. Here, HSim is $+\infty$ when either instance has an empty history, though the traditional Jaccard similarity measure will give a 0 in this case. The intuition is that the history we use for pruning is partial history in the sense that it does not contain a complete set of other instances that the given instance is similar to. Imagine we have a list of instances as shown below:

$$
I=\left\langle i_{1}, i_{2}, i_{3}, \ldots, i_{m}, i_{n}, \ldots, i_{\text {last }}\right\rangle
$$

To detect coreference relationships, we compare each instance with every other instance in the order they are placed, i.e., compare $i_{1}$ to $i_{2}, i_{3}, \ldots, i_{\text {last }}$ and then similarly compare $i_{2}$ to all other instances after it. Therefore, when we attempt to compare $i_{m}$ and $i_{n}$, each of them has only been compared with instances that are placed to the left of them and we still do not know their similarities to instances in $\left\{i_{k}\right\}(k>n)$. Due to such incomplete knowledge, an instance pair should not be pruned when seeing an empty history.

Sorting Instances. At Line 3 of Algorithm 3, we prune a pair of instances if their history similarity is below a threshold. In our algorithm, this threshold is automatically adjusted at runtime. One approach for performing such adjustment is based upon how many groundtruth coreferent pairs have already been covered by the processed instances at any given time. When more coreferent pairs have been covered, we should adopt a higher threshold in order to prune more un-coreferent pairs. This will lead to runtime savings, without the risk of missing too many true matches. However, for this approach to work, we need a sufficiently sized sample of groundtruth data, but obtaining such a sample for large-scale data is impractical.

To avoid labeling, we compute a Match Heuristic $($ MH) for each instance before executing Algorithm 3. $M H$ is 
the number of potential matches of each instance in the dataset. We sort the instances by their $M H$ in descending order, and we call Algorithm 3 on all pairs of an instance with a subsequent instance in descending $M H$ order. To compute $M H$, we treat the context of each instance as a bag of words that we call doc. For an instance, doc is extracted from all literal paths of length one, i.e., literal values from immediate triples. We then compute a cosine similarity between the $d o c$ of each instance and that of all other instances with Lucene ${ }^{2}$, a well-adopted Information Retrieval tool. We first index all the docs as documents with Lucene. Then each document is treated as a query $q$ and issued to Lucene. We count a returned document $d o c^{\prime}$ as a potential match if cosine $\left(q, d o c^{\prime}\right)$ calculated by Lucene is above a threshold $\alpha$.

A Sigmoid Function based Thresholding Approach. There could be different ways to adjust the threshold on the similarity of two instances' matching histories. The simplest way is to use a fixed threshold for the entire coreference process; however, this method may cause the system to miss a certain amount of coreferent pairs at the starting stage since we put instances with the most potential matches at the beginning of the instance list. Therefore, a thresholding approach that starts with a low value but then gradually increases the threshold could potentially be a good choice.

After sorting, each instance is associated with a match heuristic. Intuitively, the match heuristic of an instance can be considered as its weighting factor. When processing instance $i_{m}, \Sigma_{k=1}^{m-1} M H\left(i_{k}\right)$ gives the total weight of the already processed instances (i.e., their total match heuristics), indicating how important they are and thus can be helpful for thresholding.

In this paper, we propose a sigmoid function based thresholding method that utilizes such match heuristics. The original sigmoid function is defined by Equation 2:

$$
F(x)=\frac{1}{1+e^{-x}}
$$

The sigmoid function provides a continuous output with limit 1 as $x$ approaches infinity, and limit 0 as $x$ approaches negative infinity.

However, the traditional sigmoid function cannot be directly applied to our situation due to the following two aspects. First of all, the traditional function gives a value of 0.5 when $x=0$; while we require the computed threshold should be 0 when input is 0 . When $x$ is small, the entity coreference process is still in its early stage and thus we have very limited knowledge about the matching histories of the instances. Also, since we sort instances based upon the number of their potential matches in the dataset in descending order, we want to adopt a relatively low threshold at the early stage in the entire entity coreference process to reduce the risk of missing too many true matches. Furthermore, the traditional function has an upper bound of 1 . However, in our situation, we

\footnotetext{
${ }^{2}$ http://lucene.apache.org/core/3_6_0/api/core/org/apache/lucene/ search/Similarity.html
}

may not want to set such a high threshold for filtering and it would be useful if we could adjust the upper bound by setting some parameter.

Based upon the discussion above, we propose a twophased thresholding function as shown in Equation 3:

$$
\text { Thresholding }\left(i_{m}\right)= \begin{cases}0, & x \leq \sigma \\ K * F\left(x\left(i_{m}\right)-6\right), & x>\sigma\end{cases}
$$

where $\sigma$ determines when to start introducing a threshold; $K$ represents the upper bound of the computed threshold. Input $x$ is the ratio between match heuristics of already processed instances and that of all instances as computed in Eq. 4:

$$
x\left(i_{m}\right)=\frac{\sum_{k=1}^{m-1} M H\left(i_{k}\right)}{\Sigma_{k=1}^{|I|} M H\left(i_{k}\right)} * 10
$$

where $M H(i)$ is the match heuristic for instance $i$; the factor 10 enables $x$ to have the range $[0,10]$ to ensure the output (the computed threshold by Eq. 3) has the ability to transition through most of the range. We shift the original sigmoid curve to the right by replacing $x$ in Equation 2 with $x-6$, thus the computed threshold gets very close to 0 when $x=0$.

One alternative to Equation 3 is Bump, i.e., setting the history threshold to 0 at the beginning and bumping it to the upper bound when $x>\sigma$. We shall compare these different thresholding approaches in our evaluation (Section V-D).

\section{B. Evaluation Function based Context Pruning}

Another important question discussed in Section II is how to reduce the complexity of the comparison for a single pair of instances. Although an instance may have a large number of paths in its context, we should only consider those that can actually make significant contribution to the final similarity score of two instances. In this paper, we define an evaluation function in Equation 5 to judge if we should continue with the remaining paths given the similarity between two instances from their already considered paths:

$$
\operatorname{Eval}\left(s_{\text {old }}, s_{\text {curr }}\right)= \begin{cases}1, & s_{\text {curr }}-s_{\text {old }} \leq \beta \\ 0, & \text { otherwise }\end{cases}
$$

where $s_{\text {old }}$ is the similarity between two instances by considering the most highly weighted $n-1$ paths, and $s_{c u r r}$ is the similarity by considering the top $n$ paths.

Our evaluation function provides a hint of how important the remaining context is. Linking back to Algorithm 3 , at line 12, context_signif (context significance level) is initialized with $\gamma$, the maximum times we can tolerate when considering one more path does not significantly increase the similarity of two instances, i.e., $s_{c u r r}-s_{\text {old }} \leq \beta$. When this happens, Eval will return 1 and consequently at line 22, we reduce our tolerance level of such situations by 1 . When the context significance level reaches 0 , we suspect all remaining context is insignificant, and we will then decide to ignore the rest of the context to save the overall computational cost. We will show later that adopting our evaluation function can save half of the overall runtime (Section V-D). 


\section{RELATED WORK}

EPWNG [4] adopts a bag-of-paths approach to detect coreferent ontology instances. The core idea is that different properties may have quite different impact and thus for each property, a specific weight is automatically assigned. Such property weights are then combined with string matching techniques to compute instance pair similarity. Although it outperforms comparison systems on some benchmark datasets, it took about 17 hours to process 10,000 instances with dense RDF graphs. E/U-EPWNG [7] adopt context pruning techniques that are different from the evaluation function presented in this paper. They utilize sampling techniques and a utility function to prune insignificant context to scale entity coreference but do not prune dissimilar instance pairs.

Aswani et al. [2] try to match person ontology instances converted from the British Telecommunications digital library but their system needs to frequently issue search engine queries to retrieve context information. RiMOM [6], [8] is multi-strategy ontology alignment system designed for alignment at both schema and instance level. Some of the strategies include matching labels with string matching techniques and calculating cosine similarity between context of entities. AgreementMaker [9] and SERIMI [10] are another two algorithms that rely on syntactic string similarity for detecting coreferent ontology instances. Silk [11] indexes ontology instances on manually specified property values for scalability purposes and customized rules are then used to detect coreferent pairs.

$\mathrm{Hu}$ et al. [12] build a kernel by adopting the formal semantics of the Semantic Web that is then extended iteratively in terms of discriminative property-value pairs in the descriptions of URIs. Algorithms that combine formal semantics of the Semantic Web and string matching techniques also include Zhishi.me [13], LN2R [14], CODI [15] and ASMOV [16].

Instead of computing every pair of instances in a dataset, candidate selection can be adopted to select candidate instance pairs that are likely to be coreferent in order to reduce the overall search space. ASN [17] learns dynamically sized blocks for each record with a manually determined key. BSL [18] adopted supervised learning to learn a blocking scheme, a disjunction of conjunctions of (method, attribute) pairs. Cao et. al. [19] proposed a similar algorithm that utilizes both labeled and unlabeled data for learning the blocking scheme to reduce the needs of training data. PartEnum [20] is a search based algorithm that adopts a two-level partitioning and enumeration based on Hamming distance. BiTrieJoin [21] is a triebased method to support efficient edit similarity joins with sub-trie pruning. AllPairs [22] is a simple index based algorithm with certain optimization strategies. PPJoin+ [23] adopts a positional filtering principle that exploits the ordering of tokens in a record. Ed-Join [24] employed filtering methods that explore the locations and contents of mismatching n-grams. Similarly, IndexChunk [25] computes asymmetric signatures on character-level n-grams as constraints for selecting candidates. Differently, FastJoin
[26] adopts fuzzy matching techniques that consider both token and character level similarity.

\section{Evaluation}

Our system is implemented in Java and compiled with Java 6. We conduct all experiments on a RedHat machine with a 12 -core Intel $2.8 \mathrm{GHz}$ processor and 60GB memory.

\section{A. Evaluation Datasets and Metrics}

We evaluate our pruning-based entity coreference algorithm $P-E P W N G$ on two Semantic Web datasets: RKB [27] and SWAT ${ }^{3}$. For RKB, we use eight subsets: ACM, DBLP, CiteSeer, EPrints, IEEE, LAAS-CNRS, Newcastle and ECS. Our SWAT dataset consists of RDF data parsed from downloaded XML files of CiteSeer and DBLP. Although both datasets describe the academic domain and share some information, they adopt different ontologies and thus quite different predicates are involved. Also, they may have different coverage in terms of both publication date and venue. We compare on three instance categories: RKB Person, RKB Publication and SWAT Person. The groundtruth is owl:sameAs statements and can be crawled from RKB and downloaded from the SWAT website. Since the provided groundtruth was automatically derived and thus incomplete and erroneous, we manually verify and augment the groundtruth to improve their quality.

We adopt the standard metrics, including Precision: the number of correctly detected pairs divided by the total number of detected pairs given some threshold; Recall: the number of correctly detected pairs divided by the number of coreferent pairs according to the groundtruth; and F1score calculated as $2 * \frac{\text { Precision } * \text { Recall }}{\text { Precision }+ \text { Recall }}$. Furthermore, we compare the runtime of different systems. In all experiments, we collect instance context off-line and do not include this time in the reported runtime of each system.

For all experiments, we use the same parameter settings as shown in Table I. In general, adopting higher $k$ could

Table I

PARAMETER SetTings.

\begin{tabular}{|c|c|c|}
\hline Parameter & Value & Description \\
\hline$k$ (Alg. 3) & $5 \%$ & the percentage of context used for filtering \\
$\theta$ (Alg. 3) & 0.3 & if to add an instance to the history of another \\
$\gamma$ (Alg. 3) & 5 & the maximum tolerance level for context pruning \\
$\delta$ (Alg. 3) & 0.2 & the filtering threshold in Share_A_Token_Cosine \\
$\alpha$ (Sec. III-A) & 5 & sorting: whether an instance is a potential match \\
$K$ (Eq. 3) & 0.2 & the upper bound for history-based pruning \\
$\sigma$ (Eq. 3) & $30 \%$ & deciding when to apply history based pruning \\
$\beta$ (Eq. 5) & 0.1 & expected contribution of computing 1 more path \\
\hline
\end{tabular}

improve F1-score a little but slows down the process; using lower $\gamma$ causes the system to have lower F1-score because it prunes significant context; we can also gain a little higher F1-scores as we increase $\sigma$. Due to limited space, we do not present results with other parameter settings.

\section{B. Evaluation Against State-of-the-Art Systems}

We show our evaluation results in Table II. We compare $P$-EPWNG to state-of-the-art candidate selection systems: AllPairs [22], PPJoin+ [23], EdJoin [24], FastJoin [26],

${ }^{3}$ http://swat.cse.lehigh.edu/resources/data/ 
Table II

Evaluating Against State-OF-The-ARt Systems. $\mid$ Pair $s \mid$ : CAndidate Set Size; Time: The Runtime For Candidate Selection; Precision, Recall AND F1-score ARE THE RELEVANT MEASURES FOR THE ACTUAL ENTITY COREFERENCE PHASE; Total Time: THE RUNTIME FOR THE ENTIRE PROCESS, INCLUDING BOTH CANDIDATE SELECTION AND ENTITY COREFERENCE.

\begin{tabular}{|c|c|c|c|c|c|c|c|}
\hline \multirow{2}{*}{ Dataset } & \multirow{2}{*}{ System } & \multicolumn{2}{|c|}{ Candidate Selection } & \multicolumn{3}{|c|}{ Coreference } & \multirow{2}{*}{ Total Time (s) } \\
\hline & & $\mid$ Pairs & Time $(s)$ & Precision (\%) & Recall (\%) & F1-score (\%) & \\
\hline \multirow{11}{*}{ RKB Person } & P-EPWNG (Eq. 3) & 68,427 & 4.46 & 95.02 & 89.52 & 92.18 & 7.66 \\
\hline & Baseline & N/A & N/A & 95.47 & 83.66 & 89.15 & 36.69 \\
\hline & EPWNG [4] & N/A & N/A & 93.04 & 90.93 & 91.96 & $6,296.91$ \\
\hline & U-EPWNG [7] & N/A & N/A & 94.22 & 90.45 & 92.29 & 215.50 \\
\hline & Ed-Join [24] & 207,643 & 1.31 & 95.06 & 90.74 & 92.84 & 63.31 \\
\hline & AllPairs [22] & 454,972 & 0.93 & 94.26 & 90.85 & 92.52 & 83.76 \\
\hline & PPJoin+ [23] & 454,972 & 1.02 & 94.26 & 90.85 & 92.52 & 82.96 \\
\hline & FastJoin [26] & 443,846 & 3.92 & 94.27 & 90.85 & 92.52 & 81.75 \\
\hline & IndexChunk [25] & 953,300 & 2.27 & 95.03 & 90.73 & 92.82 & 149.70 \\
\hline & BiTrieJoin [21] & 969,637 & 41.92 & 95.65 & 84.61 & 89.77 & 87.66 \\
\hline & PartEnum [20] & 969,637 & 80.32 & 95.65 & 84.61 & 89.77 & 126.43 \\
\hline \multirow{11}{*}{ SWAT Person } & P-EPWNG (Eq. 3) & 43,333 & 4.50 & 99.49 & 90.88 & 94.99 & 8.61 \\
\hline & Baseline & N/A & N/A & 99.37 & 90.93 & 94.96 & 31.7 \\
\hline & EPWNG [4] & N/A & N/A & 99.45 & 90.93 & 94.99 & 16968.00 \\
\hline & U-EPWNG [7] & N/A & N/A & 99.45 & 90.93 & 94.99 & 275.17 \\
\hline & Ed-Join [24] & 226,330 & 1.56 & 99.48 & 90.81 & 94.94 & 102.77 \\
\hline & AllPairs [22] & 381,128 & 0.79 & 99.45 & 90.93 & 94.99 & 108.34 \\
\hline & PPJoin+ [23] & 381,128 & 0.86 & 99.45 & 90.93 & 94.99 & 106.72 \\
\hline & FastJoin [26] & 360,481 & 3.20 & 99.45 & 90.93 & 94.99 & 103.72 \\
\hline & IndexChunk [25] & 562,114 & 1.67 & 99.48 & 90.84 & 94.96 & 210.96 \\
\hline & BiTrieJoin [21] & 472,875 & 22.13 & 99.61 & 90.29 & 94.72 & 124.02 \\
\hline & PartEnum [20] & 473,018 & 70.47 & 99.61 & 90.29 & 94.72 & 123.10 \\
\hline \multirow{11}{*}{ RKB Publication } & P-EPWNG (Eq. 3) & 107,471 & 13.18 & 99.66 & 98.10 & 98.87 & 23.06 \\
\hline & Baseline & N/A & N/A & 98.99 & 97.28 & 98.13 & 72.1 \\
\hline & EPWNG [4] & N/A & N/A & 99.78 & 99.37 & 99.58 & 63200.71 \\
\hline & U-EPWNG [7] & N/A & N/A & 99.82 & 99.30 & 99.56 & 828.56 \\
\hline & Ed-Join [24] & $1,298,525$ & 131.85 & 99.45 & 98.36 & 98.90 & 1330.20 \\
\hline & AllPairs [22] & 581,005 & 1.41 & 99.47 & 99.06 & 99.27 & 340.14 \\
\hline & PPJoin $+[23]$ & 581,005 & 1.39 & 99.47 & 99.06 & 99.27 & 342.21 \\
\hline & FastJoin [26] & 583,839 & 75.78 & 99.47 & 99.07 & 99.27 & 430.61 \\
\hline & IndexChunk [25] & $1,594,764$ & 156.26 & 99.44 & 98.05 & 98.74 & 1242.85 \\
\hline & BiTrieJoin [21] & N/A & N/A & N/A & N/A & N/A & N/A \\
\hline & PartEnum [20] & $18,776,030$ & 3091.67 & 98.78 & 82.27 & 89.57 & 47019.50 \\
\hline
\end{tabular}

IndexChunk [25], BiTrieJoin [21] and PartEnum [20]. Although $P$-EPWNG performs both candidate selection and entity coreference, traditional candidate selection systems only do the first step. Thus, to compare to state-of-theart systems, we first run those systems on the same input to select candidate instance pairs; and then we apply the $E P W N G$ algorithm (the algorithm that does not have our proposed pruning techniques) to the selected pairs. We directly run our proposed algorithm $P$-EPWNG on the same input to get the final entity coreference results.

We also compare to $E P W N G$ [4] that performs a bruteforce pairwise comparison on all instance pairs without any pruning and to $U-E P W N G$ [7] that prunes insignificant context based upon sampling techniques and a utility function without doing candidate selection. A baseline is also tested against where we only compare name (person) and title (publication) for every pair of instances.

We randomly select $100 \mathrm{~K}$ instances for each instance category. We split each $100 \mathrm{~K}$ instances into 10 nonoverlapping and equal-sized folds, apply all algorithms on each fold and report their average. For coreference results, we report a system's best F1-score from threshold 0.1-0.9.

Baseline. The baseline is not as good as $P-E P W N G$ on F1-score and runtime. It has a much lower recall than others on RKB Person. On RKB publication, the baseline has worse precision than $P$-EPWNG since candidate selection helps to filter out some potential false positives. Although we only observed minor difference on F1-score for SWAT Person, the baseline needed significantly more time. For both RKB datasets, both $P-E P W N G$ and the baseline have worse recall than $E P W N G$, showing the need to explore context beyond just name and title and thus the need for appropriate context pruning techniques to balance runtime and F1-score. Note that the baseline requires human input on what to compare; also, in the absence of discriminative labels (such as name and title), it may not be able to achieve satisfying results.

EPWNG and U-EPWNG. Overall, compared to our previous entity coreference algorithms $E P W N G$ and $U$ $E P W N G, P-E P W N G$ achieves substantial runtime savings with slightly worse recall and F1-score. The coreference process was sped up by 2-3 orders of magnitude compared to $E P W N G$ and by a factor of 28-36 compared to $U-E P W N G$. There is no surprise that $E P W N G$ always achieves the highest recall since no pruning was adopted. Also, $P$-EPWNG achieves a little better precision on both person datasets because pruning dissimilar instance pairs can help to reduce the chance of having false positives.

The State-of-the-Art. Generally speaking, when compared to state-of-the-art candidate selection systems, $P$ $E P W N G$ selects the fewest candidate pairs and thus is able to run the fastest for the entire process, including both candidate selection and coreference. However, since $P$ $E P W N G$ is the most aggressive in pruning instance pairs, its recall and F1-score for the final coreference results are generally slightly worse than that of most of those systems.

On RKB Person, $P$-EPWNG selects 3 (EdJoin) to 13 (IndexChunk, BiTrieJoin and PartEnum) times fewer pairs 
than other systems, which leads to a speedup factor of 8 (EdJoin) to 19 (IndexChunk) on runtime but also results in $0.66 \%$ lower $\mathrm{F} 1$-score than the best (achieved by EdJoin). Although $P-E P W N G$ needs more time for candidate selection, the fact that it selects a lot fewer candidates enables it to achieve substantial runtime savings for the overall process.

We observe similar results for SWAT Person. P-EPWNG selects about 5 (EdJoin) to 13 (IndexChunk) times fewer candidates than other systems; thus the entire process runs 11 (EdJoin) to 24 (IndexChunk) times faster. Furthermore, $P$-EPWNG is also able to achieve the highest F1-score as AllPairs, PPJoin+ and FastJoin.

On RKB Publication, in addition to runtime savings, $P-E P W N G$ also achieves the highest precision among all candidate selection systems. $P-E P W N G$ prunes instance pairs most aggressively, which helps to reduce the chance of having too many false positives. Note that on this dataset, $P-E P W N G$ spends much more time selecting candidates than needed for the person datasets because our Share_A_Token_Cosine filtering is not very effective here. Share_A_Token_Cosine prunes an instance pair if they do not even share a single token in their most disambiguating context or the cosine similarity between such partial contexts is below a threshold. For person and publication instances, names and titles are generally included in the partial context used by Share_A_Token_Cosine; and it is relatively easier for titles to share a token and have a high similarity than names. So, more publication instance pairs can pass this check and are then processed by other more expensive steps.

\section{System Scalability}

We apply different systems to $100 \mathrm{~K}$ to $1 \mathrm{M}$ instances to examine their scalability. AllPairs, PPJoin+ and FastJoin achieve very similar results, so we only compare to PPJoin+. We also compare to EdJoin on both person datasets where it is better on both the number of selected pairs and the overall runtime (Table II); we did not compare to EdJoin on RKB Publication since it selects twice as many pairs as PPJoint on $10 \mathrm{~K}$ instances and thus is not expected to have better scalability. There are only $500 \mathrm{~K}$ instances in SWAT Person; also, PPJoin + does not scale to 1 million instances on RKB Person.

In Figures $2 \mathrm{a}$ to $2 \mathrm{c}, P-E P W N G$ demonstrates better scalability than the other systems; and it is about 17,5 and 11 times faster on RKB Publication, RKB Person and SWAT Person respectively on the highest compared scale. The other algorithms demonstrate a clear exponential curve; although $P-E P W N G$ is exponential, it has a much smaller exponent. Considering applying these systems to even larger datasets, the runtime differences could become even more substantial.

\section{Feature Evaluation}

We examine the effectiveness of our proposed pruning techniques by comparing to their alternatives. First, we compare different ways to adjust the threshold on instances' matching history similarity (Section III-A): Fixed (using a fixed threshold), Bump (starting with 0 and then bumping to a fixed value), Sigmoid ( $\sigma=0$ in Eq. 3) and $M$ Sigmoid ( $\sigma=30 \%$ in Eq. 3). We also consider removing our context pruning (M-Sigmoid $\backslash$ Eval) to examine its impact.

Table III

Feature Evaluation. $P$ : Precision, $R$ : Recall and $F$ : The F1-SCORE FOR $P$ AND $R ; T$ : THE RUNTIME FOR THE ENTIRE PROCESS.

\begin{tabular}{|l|l|c|c|c|c|}
\hline Dataset & System & $P(\%)$ & $R(\%)$ & $F(\%)$ & $T(\mathrm{~s})$ \\
\hline \multirow{3}{*}{ RKB } & M-Sigmoid & 95.02 & $\mathbf{8 9 . 5 2}$ & $\mathbf{9 2 . 1 8}$ & 7.66 \\
\cline { 2 - 6 } Person & Sigmoid & 95.05 & 89.22 & 92.04 & 7.56 \\
& Bump & 95.14 & 88.76 & 91.83 & 7.51 \\
& Fixed & 95.18 & 88.57 & 91.75 & $\mathbf{7 . 2 6}$ \\
\cline { 2 - 6 } & M-Sigmoid $\backslash$ Eval & $\mathbf{9 5 . 2 3}$ & 89.28 & 92.15 & 14.48 \\
\hline \multirow{4}{*}{ RKB } & M-Sigmoid & 99.66 & $\mathbf{9 8 . 1 0}$ & $\mathbf{9 8 . 8 7}$ & 23.06 \\
\cline { 2 - 6 } Publication & Sigmoid & 99.66 & 98.03 & 98.84 & 22.70 \\
& Bump & 99.66 & 97.91 & 98.78 & 22.66 \\
& Fixed & $\mathbf{9 9 . 6 6}$ & 97.80 & 98.72 & $\mathbf{2 0 . 8 6}$ \\
\cline { 2 - 6 } & M-Sigmoid $\backslash$ Eval & 99.49 & 98.09 & 98.79 & 51.00 \\
\hline \multirow{3}{*}{ SWAT } & M-Sigmoid & 99.49 & $\mathbf{9 0 . 8 8}$ & $\mathbf{9 4 . 9 9}$ & 8.61 \\
\cline { 2 - 6 } & Sigmoid & 99.49 & $\mathbf{9 0 . 8 8}$ & $\mathbf{9 4 . 9 9}$ & 8.58 \\
& Bump & 99.49 & $\mathbf{9 0 . 8 8}$ & $\mathbf{9 4 . 9 9}$ & 8.32 \\
& Fixed & $\mathbf{9 9 . 5 1}$ & 90.59 & 94.83 & $\mathbf{8 . 2 7}$ \\
\cline { 2 - 6 } & M-Sigmoid $\backslash$ Eval & 99.49 & $\mathbf{9 0 . 8 8}$ & $\mathbf{9 4 . 9 9}$ & 19.78 \\
\hline
\end{tabular}

Table III shows that M-Sigmoid has better recall than its thresholding alternatives; with similar precision, it achieves the best F1-scores. Fixed runs the fastest by using the upper bound threshold along the whole process. A twotailed t-test on the F1-scores shows: for RKB Person, the differences between M-Sigmoid and Fixed/Bump, Sigmoid are statistically significant with $\mathrm{P}$ values of 0.0001 and 0.0004; for RKB Publication, the differences between $M$ Sigmoid and Fixed/Bump, Sigmoid are significant with $\mathrm{P}$ values of 0.0001 and 0.0101. Furthermore, M-Sigmoid is about 1.9 to 2.2 times faster than M-Sigmoid $\backslash$ Eval, showing the effectiveness of our evaluation function.

\section{CONCLUSION}

In this paper, we propose $P-E P W N G$, a pruning-based algorithm to scalably detect coreference relationships in the Semantic Web. To reduce the impact of number of instances on runtime, an on-the-fly candidate selection technique is proposed for filtering un-coreferent instance pairs if their matching histories are not sufficiently similar. A sigmoid function based thresholding method is also proposed to balance F1-score and runtime. To speed up the computation for a single pair of instances, we further propose an evaluation function to prune insignificant context to their final similarity score. We compare $P$ $E P W N G$ to 9 state-of-the-art candidate selection and entity coreference algorithms and show that our system runs 18 to 24 times faster while only making a small sacrifice in F1-scores $(0.71 \%$ at maximum) for the final coreference results. Also, a scalability test on up to $1 \mathrm{M}$ instances shows runtime savings of 16-370 hours, depending on the dataset. Finally, a feature evaluation verifies the effectiveness of each proposed pruning technique compared to alternatives. For future work, we will apply $P-E P W N G$ to datasets from the Ontology Alignment Evaluation Initiative ${ }^{4}$ and also evaluate if our pruning techniques can help to scale other entity coreference algorithms [8], [15].

\footnotetext{
${ }^{4}$ http://oaei.ontologymatching.org
} 


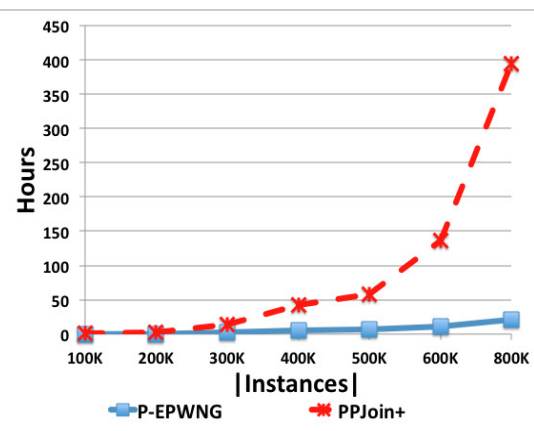

(a) RKB Publication

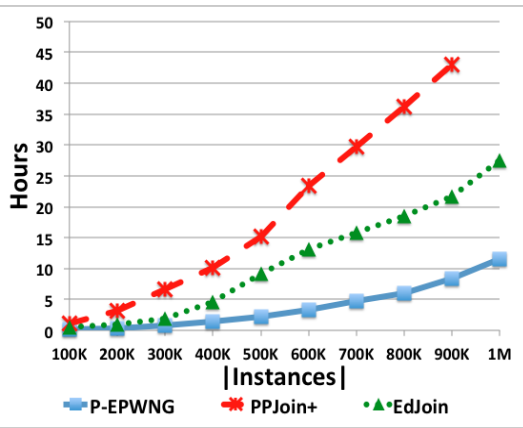

(b) RKB Person

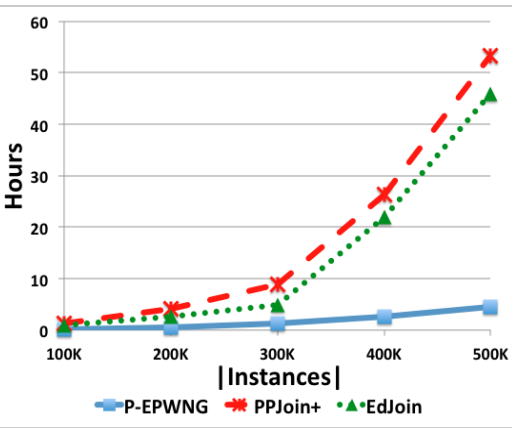

(c) SWAT Person

Figure 2. System Scalability

\section{ACKNOWLEDGMENT}

This project was partially sponsored by the U.S. Army Research Office (W911NF-11-C-0215). The content of the information does not necessarily reflect the position or the policy of the Government, and no official endorsement should be inferred.

\section{REFERENCES}

[1] C. Bizer, T. Heath, and T. Berners-Lee, "Linked data - the story so far," Int. J. Semantic Web Inf. Syst., vol. 5, no. 3, pp. 1-22, 2009.

[2] N. Aswani, K. Bontcheva, and H. Cunningham, "Mining information for instance unification," in International Semantic Web Conference (ISWC), 2006, pp. 329-342.

[3] J. Hassell, B. Aleman-Meza, and I. B. Arpinar, "Ontologydriven automatic entity disambiguation in unstructured text," in International Semantic Web Conference, 2006, pp. $44-57$.

[4] D. Song and J. Heflin, "Domain-independent entity coreference for linking ontology instances," ACM Journal of Data and Information Quality (ACM JDIQ), 2012.

[5] H. Halpin, P. J. Hayes, J. P. McCusker, D. L. McGuinness, and H. S. Thompson, "When owl: sameAs isn't the same: An analysis of identity in linked data," in 9th International Semantic Web Conference (ISWC), 2010, pp. 305-320.

[6] J. Li, J. Tang, Y. Li, and Q. Luo, "RiMOM: A dynamic multistrategy ontology alignment framework," IEEE Trans. Knowl. Data Eng., vol. 21, no. 8, pp. 1218-1232, 2009.

[7] D. Song and J. Heflin, "A pruning based approach for scalable entity coreference," in Proceedings of the TwentyFourth International Florida Artificial Intelligence Research Society Conference (FLAIRS), 2012, pp. 98-103.

[8] Z. Wang, X. Zhang, L. Hou, Y. Zhao, J. Li, Y. Qi, and J. Tang, "RiMOM results for OAEI 2010," in Proceedings of the 5th International Workshop on Ontology Matching, 2010.

[9] I. F. Cruz, F. P. Antonelli, and C. Stroe, "AgreementMaker: Efficient matching for large real-world schemas and ontologies," $P V L D B$, vol. 2, no. 2, pp. 1586-1589, 2009.

[10] S. Araújo, A. P. de Vries, and D. Schwabe, "SERIMI results for OAEI 2011," in Proceedings of the 6th International Workshop on Ontology Matching (OM), 2011.

[11] J. Volz, C. Bizer, M. Gaedke, and G. Kobilarov, "Discovering and maintaining links on the web of data," in 8th International Semantic Web Conference (ISWC), 2009, pp. $650-665$.

[12] W. Hu, J. Chen, and Y. Qu, "A self-training approach for resolving object coreference on the semantic web," in Proceedings of the 20th International Conference on World Wide Web (WWW), 2011, pp. 87-96.
[13] X. Niu, X. Sun, H. Wang, S. Rong, G. Qi, and Y. Yu, "Zhishi.me - weaving Chinese linking open data," in International Semantic Web Conference, 2011, pp. 205-220.

[14] F. Saïs, N. Pernelle, and M.-C. Rousset, "Combining a logical and a numerical method for data reconciliation," Journal on Data Semantics XII, vol. 12, pp. 66-94, 2009.

[15] J. Noessner, M. Niepert, C. Meilicke, and H. Stuckenschmidt, "Leveraging terminological structure for object reconciliation," in 7th Extended Semantic Web Conference (ESWC), 2010, pp. 334-348.

[16] Y. R. Jean-Mary, E. P. Shironoshita, and M. R. Kabuka, "Ontology matching with semantic verification," Journal of Web Semantics, vol. 7, no. 3, pp. 235-251, 2009.

[17] S. Yan, D. Lee, M.-Y. Kan, and C. L. Giles, "Adaptive sorted neighborhood methods for efficient record linkage," in ACM/IEEE Joint Conference on Digital Libraries (JCDL), 2007, pp. 185-194.

[18] M. Michelson and C. A. Knoblock, "Creating relational data from unstructured and ungrammatical data sources," J. Artif. Intell. Res., vol. 31, pp. 543-590, 2008.

[19] Y. Cao, Z. Chen, J. Zhu, P. Yue, C.-Y. Lin, and Y. Yu, "Leveraging unlabeled data to scale blocking for record linkage," in Proceedings of the 22nd International Joint Conference on Artificial Intelligence (IJCAI), 2011, pp. 2211-2217.

[20] A. Arasu, V. Ganti, and R. Kaushik, "Efficient exact setsimilarity joins," in Proceedings of the 32nd International Conference on Very Large Data Bases (VLDB), 2006, pp. 918-929.

[21] J. Wang, G. Li, and J. Feng, "Trie-join: Efficient triebased string similarity joins with edit-distance constraints," PVLDB, vol. 3, no. 1, pp. 1219-1230, 2010.

[22] R. J. Bayardo, Y. Ma, and R. Srikant, "Scaling up all pairs similarity search," in Proceedings of the 16th International Conference on World Wide Web, 2007, pp. 131-140.

[23] C. Xiao, W. Wang, X. Lin, J. X. Yu, and G. Wang, "Efficient similarity joins for near-duplicate detection," ACM Trans. Database Syst., vol. 36, no. 3, p. 15, 2011.

[24] C. Xiao, W. Wang, and X. Lin, "Ed-join: an efficient algorithm for similarity joins with edit distance constraints,' Proc. VLDB Endow., vol. 1, no. 1, pp. 933-944, 2008

[25] J. Qin, W. Wang, Y. Lu, C. Xiao, and X. Lin, "Efficient exact edit similarity query processing with the asymmetric signature scheme," in Proceedings of the ACM SIGMOD International Conference on Management of Data, 2011, pp. 1033-1044.

[26] J. Wang, G. Li, and J. Feng, "Fast-join: An efficient method for fuzzy token matching based string similarity join," in Proceedings of the 27th International Conference on Data Engineering (ICDE), 2011, pp. 458-469.

[27] H. Glaser, I. Millard, and A. Jaffri, "RKBExplorer.com: A knowledge driven infrastructure for linked data providers," in The 5th European Semantic Web Conference (ESWC), 2008, pp. 797-801. 